\title{
Evolution in the formation of graphene nanocapsules from coal tar pitch
}

\author{
D. M. Puente-Siller ${ }^{1}$ (D) A. E. García-Castillo ${ }^{1}$ (D) J. A. López-Corpus ${ }^{1}$ (D) \\ A. Perea-Garduño ${ }^{1}$ (B)
}

Received: 5 October 2019/Revised: 7 February 2020/Accepted: 13 March 2020/Published online: 5 April 2020

(C) The Author(s) 2020

\begin{abstract}
It has recently been reported that coal tar pitch (CTP) can be utilised as raw material for the production of graphene nanocapsules (GNCs) because it is formed by a great quantity of aromatic organic compounds (which promote the rearrangement of double bonds by a process of polymerisation). Due to the importance of graphene and the search for a non-expensive methodology to produce it, this work used CTP to synthesise GNCs using an in situ activation technique at low temperatures and evaluating the effect of the working temperature on the formation of such nanostructures. In other words, analysing the form of the particle as the temperature rises from 600 to $900{ }^{\circ} \mathrm{C}$. As result of the experimentation, powders were obtained and analysed by the techniques of X-Ray Diffraction, Raman Spectroscopy and Microscopy, employing Field Emission Scanning Electron Microscopy by normal mode as well as by Scanning Transmission Electron Microscopy and a High-Resolution Scanning Electron Microscopy. The results show that working with temperatures between 800 and $850{ }^{\circ} \mathrm{C}$ promotes the production of GNCs, considering that their size reduces as the working temperature rises.
\end{abstract}

Keywords Synthesis $\cdot$ Nanocapsule $\cdot$ Graphene $\cdot$ Tar $\cdot$ Agglomeration

\section{Introduction}

Coal tar is a valuable chemical material from which compounds that contain nitrogen are mainly separated, such as indole, carbazole, pyridine and quinoline, however, more than 500 chemicals have been identified. There are non-catalytic processes that allow the separation of

A. E. García-Castillo

adriana_eloisagc@hotmail.com

D. M. Puente-Siller

dmpuentes@gan.com.mx

J. A. López-Corpus

jalopezc@gan.com.mx

A. Perea-Garduño

aperea@gan.com.mx

1 Department of Research and Development, Altos Hornos de México, S. A. B. de C. V, Prolongación Juárez, S/N, La Loma, 25770 Monclova, Coahuila, Mexico nitrogenous compounds from coal tar as alkaline fusion (with potassium hydroxide) (Zhang et al. 2017). The distillation residue, which is obtained during the refinement of the industrial coal tar with an output between 50\% and 55\% of the weight, in relation to the crude tar, is called coal tar pitch (CTP) (Zander 1995).

Coal tar pitch (CTP) is a material with high aromaticity which is defined as the relation of present carbon in the aromatic compounds with respect to total carbon. It has been concluded that the high molecular weight portion of the pitch is principally a mixture of heterocyclic systems instead of a mixture of polycyclic aromatic hydrocarbons (PAH), it has been estimated that the high molecular weight heterocyclic portion of the pitch amounts to approximately $10 \%-15 \%$ of the weight in relation with the complete pitch (Zander 1995). In other words, CTPs are made of compounds of aromatic nature which are susceptible to be transformed, by controlled thermal processes, in graphite or graphite-like structures, which turn CTPs into 
excellent precursors of carbon materials. The chemicalstructural complexity of CTPs gives way for variations in the operational conditions of these processes (named carbonisation) to generate materials with different structures and properties (Granada 2011).

The pyrolysis of CTP involves the dehydrogenative polymerisation of the aromatic units, which occurs with the simultaneous distillation of light compounds, leading to the formation of bigger plane macromolecules. The CTP compounds with low molecular weight have an important role in the pyrolysis process because they are eliminated or partially incorporated into the CTPs systems, depending on experimental conditions (Bermejo et al. 1995).

The array of carbon materials that present mixtures of the three types of hybridisation include numerous materials with practical interest, named carbon forms, and with the exception of diamond-type amorphous carbon, they present a predominant proportion of carbon atoms in hybridisation $\mathrm{sp}^{2}$. Because of this, it is a known fact that the structure of graphite widely dominates the discussions regarding science and technology of carbon materials. In this context, it is common to consider the majority of carbon forms (CTP, activated carbon, pyrolytic carbon, type glass carbon, coke, carbon fibres, carbonaceous mesophase, carbon blacks) as related with the graphite structure that is formed as reference to the maximum grade of structural perfection, having been established as criteria for being able to consider the carbon material as graphite (or more precisely, graphitic material), that within itself the graphenes are arranged in parallel between them in a three-dimensional (3D) crystalline network (Tascón 2007).

\subsection{Graphene: definition, properties and applications}

According to Rodríguez-González and Vasilievna-Kharissova (2008), graphene is a bidimensional nanometric structure of atoms with strong cohesion in a uniform surface, slightly flat, with rippling of an atom of similar thickness and appearance to a honeycomb. This material can have one or up to ten overlapping layers and its properties are a function of its dimensionality, thus, it can be classified in three types: monolayer, double-layer and those which are between 3 to 10 layers, called multilayer.

Graphene stands out due to its good electrical, structural, impermeable, physical, chemical and thermic conductivity properties (consequence of its network's structure) and therefore, can be applied to a great variety of sectors (Alcolea-Sánchez 2013). In other words, it can be utilised in the development or improvement of technology (SaldivarLarré 2014).

The preparation of nanoparticles of graphene (GNs) includes methods such as epitaxial growth, arc discharge, graphene oxide chemical reduction, thermal exfoliation of graphene compounds, liquid-phase exfoliation and chemical vapour deposition. Nevertheless, there are difficulties because of being a small-scale preparation and due to complex processes or elevated costs, which restrict the wide applications of GNs. Therefore, it is necessary to produce a simple method to create GNs for industrial use. As a solution, CTP can be thought as a cheap organic material which results from tar and can be used to produce GNs (by a pyrolysis process), considering that the morphology of CTP is a primary factor of the microstructure of the resulting carbon material (Xu et al. 2014). Depending on the composition of coal tar (determined by the process used to produce coke) and the conditions of distillation, two distinct types of CTP can be commercialised: binder and impregnating pitches (Fernández-García et al. 2017).

On the other hand, besides GNs, graphene can be found as nanocapsules (GNCs) which have a multilayer 3D structure and can be produced at low temperatures (Yoon et al. 2012). Particularly, 3D graphene structures can be produced in various substrates as nanoparticles, non-metal porous structures, metal foams (Olszowska et al. 2017).

Molecules of polycyclic aromatic compounds can be found in CTP, which by using a metal oxide agent and in situ activation agent, are used as base for the construction of the 3D interconnected networks of graphene. Therefore, CTP can be treated to form a great quantity of active polycyclic radicals that act as a construction block and make its polymerisation and aromatisation possible, favouring an interconnected 3D polymer that will transform into GNCs once it is thermally treated (He et al. 2017a). This route can become a general synthetic way to synthesise other 3D graphene materials (He et al. 2017b).

There is great interest in the analysis of the synthesis process of GNCs. For example, Yoon et al. (2012), used nanoparticles of nickel as substrate in the formation of these materials, employing a technique of polyol carburisation and afterwards, giving thermal treatment to the synthesised particles. The authors analysed the solids by XRD and Transmission Electron Microscope (TEM) in situ, which gave them the opportunity to study the synthesis as time went by. On the other hand, Olszowska et al. (2017) elaborated an extensive revision about 3D nanostructured graphene, which considered nanoshells, encapsulates, foams among others and employing the hydrothermal method and chemical vapour deposition. However, an analysis of the evolution of nanoparticles as the working temperature increases to undertake a thermosynthesis with in situ activation has not been found up to today.

Due to the latter, the objective of this research is to analyse the synthesis process for the procurement of GCNs based on the reported technique by $\mathrm{He}$ et al. (2017b), 
varying the working temperature from 600 to $900{ }^{\circ} \mathrm{C}$ in order to study the process of the particle's transformation, as it is described in Sect. 2

\section{Materials and methods}

The technique reported by $\mathrm{He}$ et al. (2017b), is to crush and mix $4.2 \mathrm{~g}$ CTP, $16.8 \mathrm{~g}$ nano- $\mathrm{ZnO}$ and $6.0 \mathrm{~g} \mathrm{KOH}$ in solid state. The pulverised mixture was transferred to a corundum pot, which was placed in a horizontal tubular oven and heated up to $150{ }^{\circ} \mathrm{C}$ at a rate of $5{ }^{\circ} \mathrm{C} / \mathrm{min}$ with a residence time of $30 \mathrm{~min}$. Subsequently, it was heated to $800{ }^{\circ} \mathrm{C}$ for $1 \mathrm{~h}$ in a stream of nitrogen with a flow rate of $60 \mathrm{~mL} / \mathrm{min}$.

In the present work, all of the used reagents were analytical grade; $1.6 \mathrm{~g}$ CTP (-100\#), $6.4 \mathrm{~g}$ nano-ZnO (SigmaAldrich) and $2.8 \mathrm{~g} \mathrm{KOH}$ (FagaLab) were pulverised and mixed together. The mixture was transferred to an alumina crucible and was introduced into a horizontal oven. Since the beginning of the experiment, there was a nitrogen flow of $60 \mathrm{~mL} / \mathrm{min}$ and each test was submitted to two heating ramps (varying the final temperature according to the experiment in progress). The analysed temperatures were $600,700,800,850$ and $900{ }^{\circ} \mathrm{C}$. The initial heating cycle consisted in the increase of temperature up to $150{ }^{\circ} \mathrm{C}$ at a rate of $10{ }^{\circ} \mathrm{C} / \mathrm{min}$ with a residence time of $30 \mathrm{~min}$. The second ramp achieved the working temperature at the same rate as the first, however, the time of residence was of $1 \mathrm{~h}$. Once the experiment was finalised, the obtained solids were washed with hydrochloric acid ( $2 \mathrm{M})$ and rinsed using distilled water. Lastly, they were left to dry in a drying stove for $24 \mathrm{~h}$ at $110^{\circ} \mathrm{C}$.

\subsection{Analysis}

CTP and the obtained solids were analysed for characterisation. Table 1 shows the analysis for each sample.

It is important to note that besides what is described in Table 1, the ash content in the CTP was determined using the norm ASTM-D2415-98. Also, the rest of the analysis were determined by IP: ASTM-D92-18; SP: ASTM-D3614/D36M-09; IT: ASTM-D4312-95a; IQ: ASTM-D231898 and CV: ASTM-D4715-98.

The details of the performed analysis are described next. The TGA was analysed using a TA INSTRUMENTS SDT Q600 V20.9 Build 20. A heating rate of $10^{\circ} \mathrm{C} / \mathrm{min}$ was used to increase the temperature from 25 to $1000{ }^{\circ} \mathrm{C}$ in a nitrogen atmosphere; the XRD was carried out in a Panalitical XpertPro diffractometer equipped with a cobalt tube. The scan was done from 0 to $110^{\circ}$ employing High Score Plus software to identify the phases. For the GC/MS analysis, a Shimadzu CGMS-QP2010 SE was used, the column Rxi-5Sil MS was of $30 \mathrm{~m}$ and $0.25 \mathrm{~mm}$ of interior diametre, with a temperature of the ion source of $200{ }^{\circ} \mathrm{C}$ and the interface temperature was $280{ }^{\circ} \mathrm{C}$. In the microstructure analysis a FEI microscope, Scios model, was utilised. The images were taken in STEM and normal modes, additionally, a FESEM model 7800 Prime from JEOL was used. Lastly, the Raman Spectrometry was carried out in an Oriba confocal microscope Xplora model with target at 10 and $50 x$, Gratin $600,25 \%$ to $50 \%$ energy and a working range between 100 to $4000 \mathrm{~cm}^{-1}$.

\section{Results and discussions}

The CTP sample has a $2.48 \%$ of ash, therefore, its carbon content is considerably high $(97.52 \%)$. In regard to the characterisation of the sample, it was determined that its ignition and softening points are 250 and $109.8{ }^{\circ} \mathrm{C}$, respectively. It has a $42.8 \%$ of toluene insoluble and $20.8 \%$ of quinoline insoluble components. Moreover, it has a coking value of $61.3 \%$.

On the other hand, it was possible to identify the components in the CTP by GC/MS. The results show the presence of compounds such as acenaphtene, anthracene, $4 \mathrm{H}$ - cyclopenta[def]phenanthrene, $11 \mathrm{H}$-benzo[b]fluorene, benzo[ghi] fluoranthene, benzo(b)carbazole, benzo(a)anthracene-7-carbonitrile, 9H-ciclopenta[a]pyrene, ciclohexanepropanoic acid, 2-propenyl ester; cyclohepta[e]1,2-

Table 1 Analysis for tar and CTP samples

\begin{tabular}{lllllllllll}
\hline Sample & Analysis & & & & & & \\
\cline { 2 - 9 } & TGA/DSC* & XRD* & GC/MS* & IP* & SP* & IT* & IQ* & CV $^{*}$ & $\mathrm{M}^{*}$ & $\mathrm{RS}^{*}$ \\
\hline CTP & $\mathrm{Y}$ & $\mathrm{Y}$ & $\mathrm{Y}$ & $\mathrm{Y}$ & $\mathrm{Y}$ & $\mathrm{Y}$ & $\mathrm{Y}$ & $\mathrm{Y}$ & $\mathrm{Y}$ & $\mathrm{Y}$ \\
Synthesised solids & $\mathrm{N}$ & $\mathrm{Y}$ & $\mathrm{N}$ & $\mathrm{N}$ & $\mathrm{N}$ & $\mathrm{N}$ & $\mathrm{N}$ & $\mathrm{N}$ & $\mathrm{Y}$ & $\mathrm{Y}$ \\
\hline
\end{tabular}

*TGA/DSC thermal gravimetric analysis/differential scanning calorimetry; XRD X-ray diffraction; GC/MS gas chromatography/mass spectrometry; $I P$ ignition point; $S P$ softening point; $I T$ insoluble in toluene; $I Q$ insoluble in quinoline; $C V$ coking value; $M$ microstructure analysis (STEM scanning transmission electron microscopy (FEI); FESEM field emission scanning electron microscopy); RS: Raman spectroscopy; Y indicates samples being undertaken analysis, $\mathrm{N}$ indicates sample not being undertaken analysis 
oxazine, ciclohexanecarboxamida, N-furfuryl, benzo[c]phenanthrene, benzo[e]acefenantrilene, benzo[j]fluoranthene, benzo[ghi]perylene, benzo[a]anthracene, pyrene, triphenylene and naphthalene, among others in very low percentages.

Figure 1 shows the results of TGA/DSC analysis, where the phase changes related to the polymerisation of the sample can be seen, as well as the loss of components. This figure shows some interesting information. At low temperatures (close to $50{ }^{\circ} \mathrm{C}$ ), a phase change exists that can be related to the polymerisation of the sample. This probably corresponds to the physical changes derived from the fusion process. In other words, CTP has a softening point at $109.8^{\circ} \mathrm{C}$, however, the heat absorption has to start earlier and it is precisely this fact which is being referred to on the observed energy consumption at $50{ }^{\circ} \mathrm{C}$. In the other hand, after an increase in the heat flow, a constant reduction of it begins, which can be associated with an evaporation of the sample. In connection to this, the resulting curve of the TGA analysis can be seen, in which a loss of weight exists starting at $300{ }^{\circ} \mathrm{C}$. This transformation can be related to the evaporation of phases that make the sample. It is important to mention that this phenomenon matches with the phase change presented in the DSC curve. Another interesting point is that starting at approximately $550{ }^{\circ} \mathrm{C}$, there is not a significant loss in mass, by which it could be understood that this condition favours the polymerisation process to form desired GNCs. Said mass loss at temperatures lower than $550^{\circ} \mathrm{C}$ can be reinforced by analysing the components of CTP, where the presence of aromatic compounds with boiling points below $550{ }^{\circ} \mathrm{C}$ is disclosed.

Therefore, it could be said that during the synthesis process of GNCs, initially, a loss in humidity and the softening of the pitch is triggered and at temperatures above $600{ }^{\circ} \mathrm{C}$, the process of polymerisation is promoted.

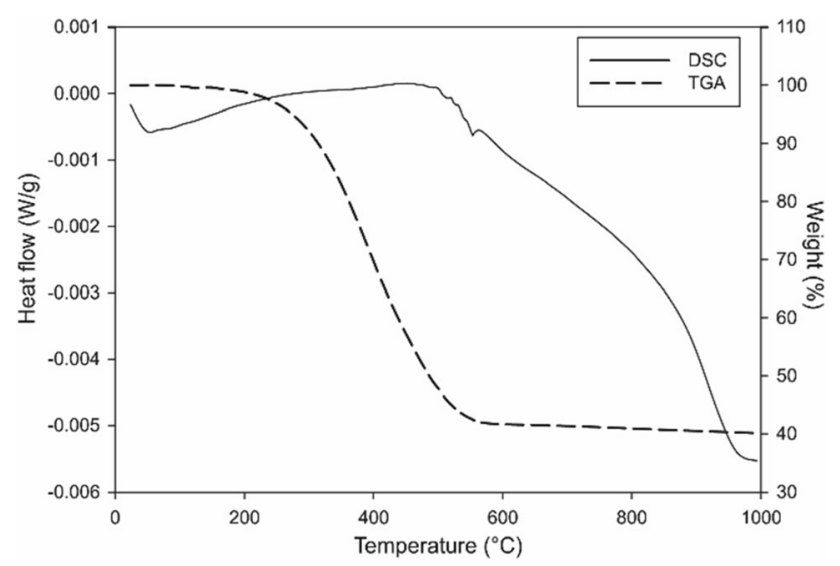

Fig. 1 TGA/DSC results for CTP
The results collected from the chemical analyses performed to the synthesised powders at different temperatures are described next. It is worth mentioning that each sample was washed and rinsed in the same way. Similarly, the drying temperature and time were exactly the same, consequently, the difference in the results is due to the characteristics of each solid.

The results from XRD pertinent to the synthesised solids at different temperatures are shown in Fig. 2. As it can be seen, a comparison between the crystallinity of CTP without heat treatment and the synthesised solids at different temperatures is done. In the mentioned image, it can be observed that at 600 and $700{ }^{\circ} \mathrm{C}$ it is not possible to remove all of the zinc oxide, while in the case of the treatments at 800 and $850{ }^{\circ} \mathrm{C}$, a particle with low crystallinity can be seen as reported by He et al. (2017b) with a very different diffraction pattern to that displayed by the CTP before beginning with the experimentation (which can be viewed in Fig. 2). Lastly, in the case of the particle synthesised at $900{ }^{\circ} \mathrm{C}$, it can be noted it is not a crystalline particle, nevertheless, it does present defined diffraction patterns, slightly shifted in relation to the solids at 600 and $700{ }^{\circ} \mathrm{C}$. It is believed that this behaviour is clearly linked with the working temperature, under which the nanocapsule is not as permeable and it hampers the washing with acid for the removal of zinc oxide.

Figures 3 and 4 show the results of RS analysis done to samples which were treated at 600 and $700{ }^{\circ} \mathrm{C}$ (as evidence of the low zinc oxide removal at lower temperatures). Such analysis were done using different focuses on the same sample. Furthermore, in both cases it initiates with the presence of vibrations in the zone of inorganic compounds and even though both samples also exhibit the corresponding vibration of bands $G$ and $D$ of a graphene material, in the greater wavelengths there are considerably

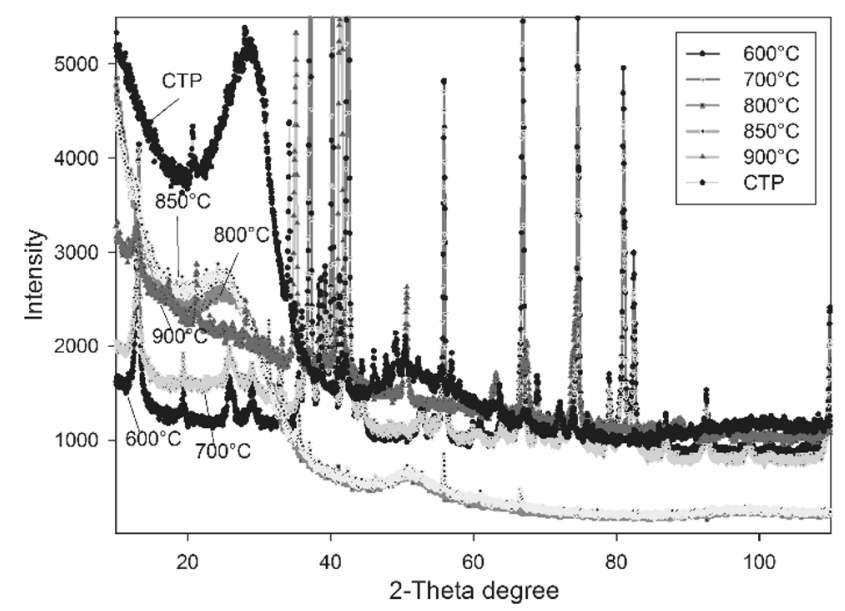

Fig. 2 XRD results for CTP sample and obtained solids at different working temperatures 


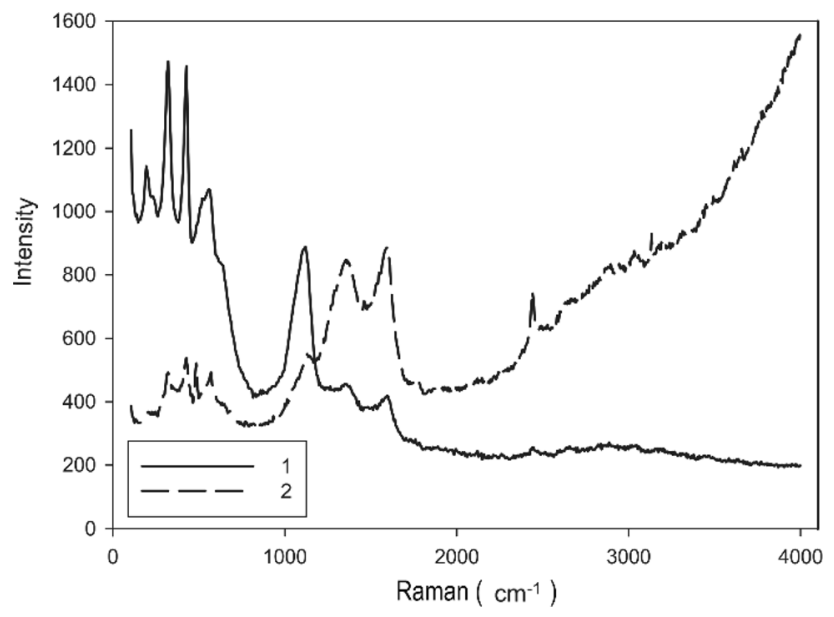

Fig. 3 RS results for a sample of solids obtained at $600{ }^{\circ} \mathrm{C}$

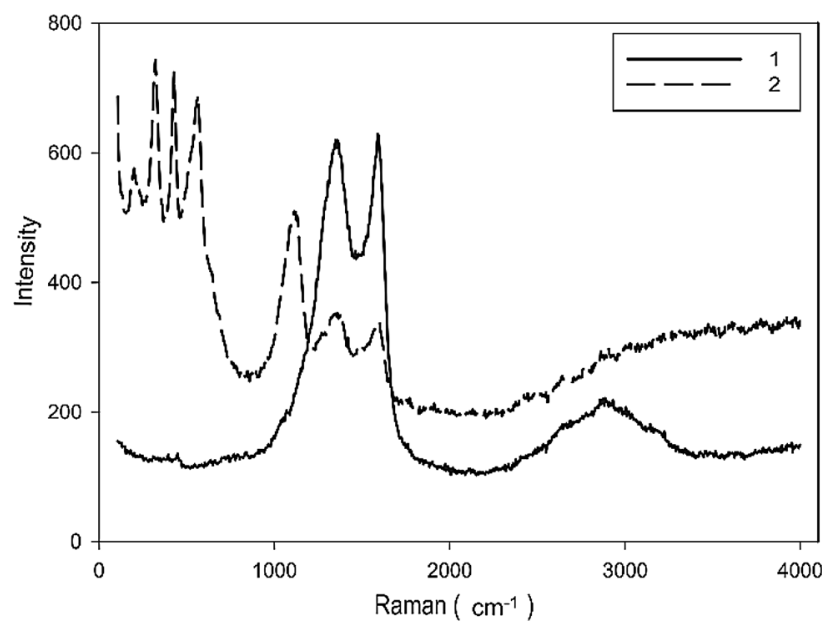

Fig. 4 RS results for a sample of solids obtained at $700{ }^{\circ} \mathrm{C}$

high vibrations (especially in the case of particles synthesised at $600{ }^{\circ} \mathrm{C}$ ), which can lead to believe that there is still a great quantity of organic compounds that did not modify their structure to form GNCs. These figures also reference the heterogeneity of the samples.

Figure 5 is found to be in the same context, where a comparison between synthesised particles and the initial material (pitch) is done. As it can be seen, the pitch does not show vibrations in the zone of inorganics, however, it does present them in the zone of double carbon bond as well as in the rest of the organic compounds. The vibrations are so intense that those obtained in the synthesised material cannot be completely identified, therefore, a closeup of the zone with least intensity is shown in Fig. 6 in order to analyse the process of synthesis at different temperatures. As it can be observed, all of the solids have vibrations in bands $\mathrm{D}$ and $\mathrm{G}\left(\approx 1326-1592 \mathrm{~cm}^{-1}\right)$, however, they have different intensities and not all meet with the vibration of band $2 \mathrm{D}$, which is the necessary vibration

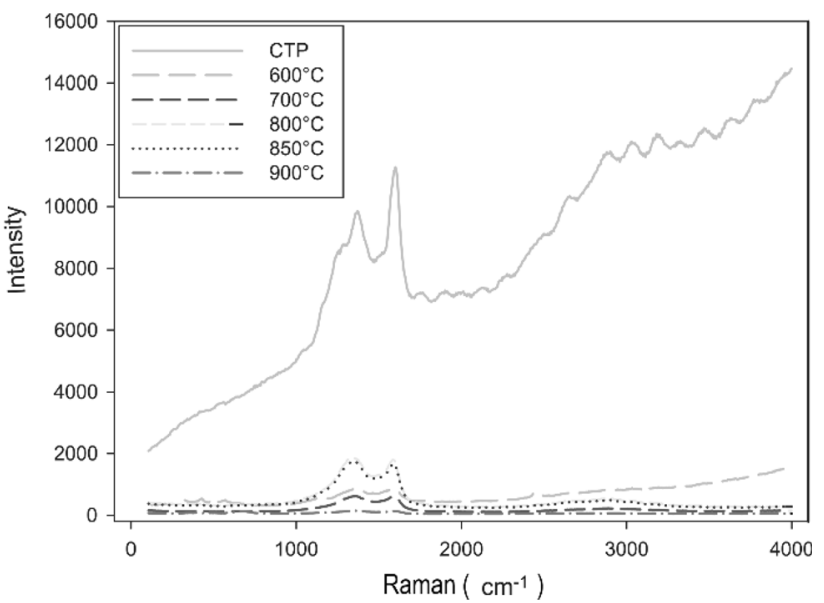

Fig. 5 RS results for a sample of CTP and solids obtained at different working temperatures

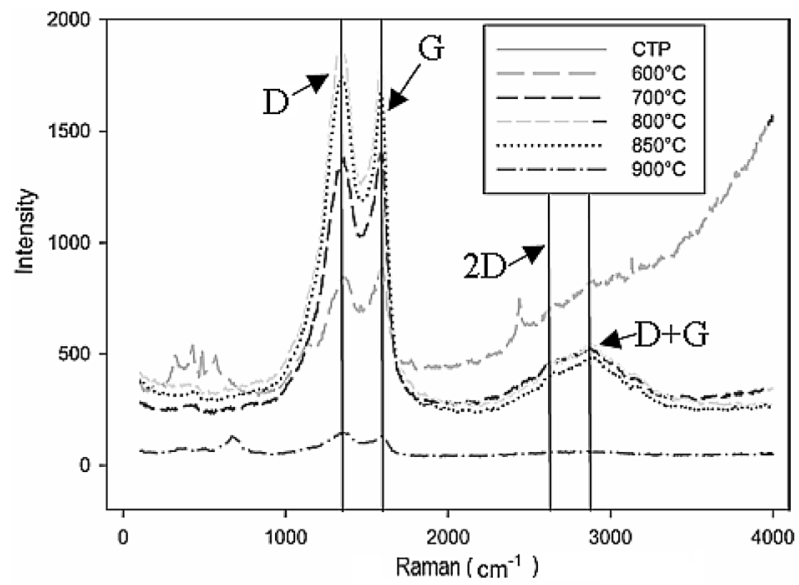

Fig. 6 Comparison of RS results for samples of solids obtained at different working temperatures

for the identification of a graphene material (He et al. $2017 b$ ) and which was found in this study at approximately $2660 \mathrm{~cm}^{-1}$. In the case of the synthesis carried out at $600{ }^{\circ} \mathrm{C}$ and as mentioned before, there is presence of organic and inorganic compounds, however, when working at $700{ }^{\circ} \mathrm{C}$, the three characteristic vibrations of graphene can be found (without putting aside the presence of inorganic material shown in Fig. 4), additionally, such vibrations are intensified with the increase of temperature up to 800 and $850{ }^{\circ} \mathrm{C}$ as these samples have a better band definition and greater intensity than the rest of the working temperatures. Furthermore, said vibrations match with the reports by $\mathrm{He}$ et al. (2017b). The latter proposes that as temperature increases, it is possible to obtain a more defined graphene material, whose bands contain greater vibrations in the characteristic wavelengths, although, this does not present when working at $900{ }^{\circ} \mathrm{C}$ as bands $\mathrm{D}$ and $\mathrm{G}$ can barely been seen in the sample and band 2D is not there (at least not at a noticeable intensity to be compared with 


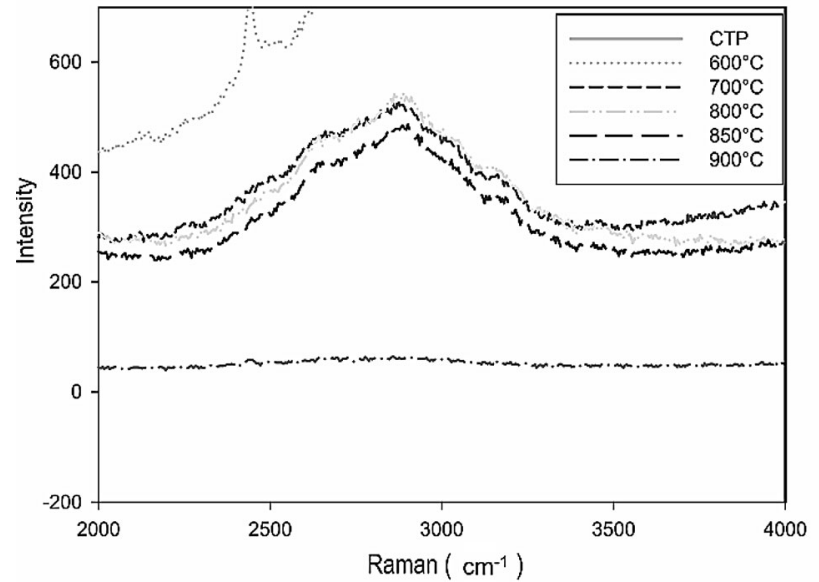

Fig. 7 2D and D + G bands obtained from RS results for a sample of solids obtained at different working temperatures

the rest of the solids), nevertheless, it contains inorganic materials (vibration in low wavelengths). Considering that all samples were processed under the same conditions (washing, rinsing and drying), it can be said that as a consequence of the working temperature, it was not possible to completely wash the microstructure of the obtained particle or that the polymerisation of organic compounds did not happen as expected, diminishing the quality of the particle.

On the other hand, Fig. 7 specifically shows the zone between 2000 and $4000 \mathrm{~cm}^{-1}$ in order to thoroughly analyse the vibrations in this wavelength. The presence of a spike activated by defects called $\mathrm{D}+\mathrm{G}$ can be seen in the figure, which are visible near the $2900 \mathrm{~cm}^{-1}$ (Jibrael and Mohammed 2016) and that are presented in this case under the wavelength of $2899 \mathrm{~cm}^{-1}$, in solids treated between 600 and $850{ }^{\circ} \mathrm{C}$.

It has been mentioned earlier that when comparing the RS results of the particle synthesised at $900{ }^{\circ} \mathrm{C}$ with the others, it was not possible to clearly observe the vibrations which correspond to a graphene material, however, if Fig. 8 is observed, it is possible to see that when working at $900{ }^{\circ} \mathrm{C}$ the synthesised solids can have vibrations in the bands which are characteristic of those found in graphene (with intensities much lower than the obtained at 800 and $850^{\circ} \mathrm{C}$ ). In Fig. 8, the results of the mathematical deconvolution analysis done to the patterns at 800,850 and $900{ }^{\circ} \mathrm{C}$ are shown. This data analysis was done using Origin 9.0 software. It is noteworthy that the results of the synthesised solids are not presented. This is because the working temperature proposed in the literature starts at $800{ }^{\circ} \mathrm{C}$ and furthermore, due to the heterogeneity of the sample, a high error range is considered in the analysis of the Raman pattern (due to the significative presence of inorganic material, which can be observed in Figs. 3 and 4).

According to Silva-Molina (2016), the analysis of the obtained experimental results of bands $G$ and $2 D$, after deconvolution of Raman patterns, were correlated to the number of layers of graphene material. In other words, the ratio of intensities of band $2 \mathrm{D}$ with respect to band $\mathrm{G}$
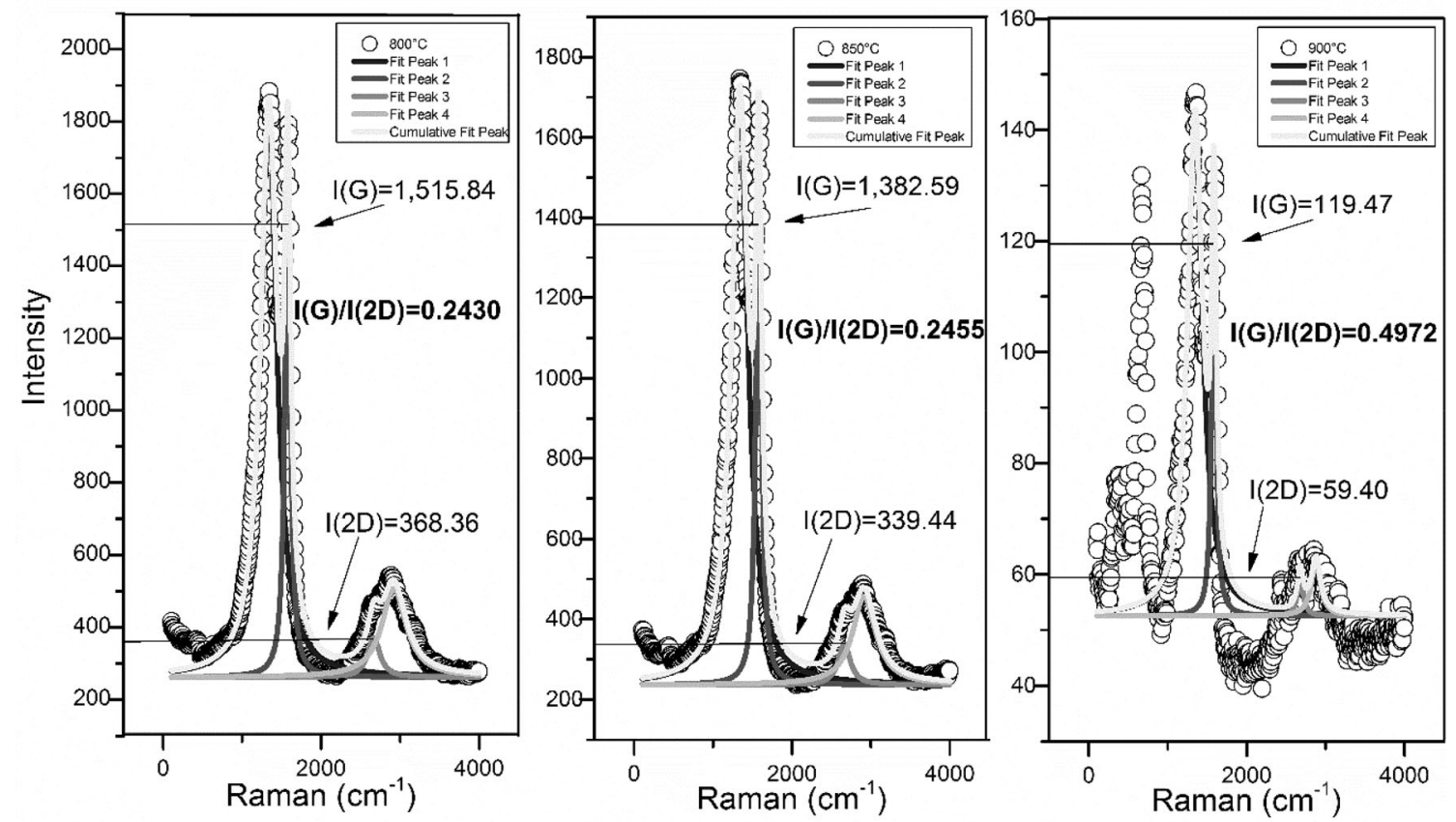

Fig. 8 Comparative of RS results for the sample of solids obtained at temperatures of 800,850 and $900{ }^{\circ} \mathrm{C}$ and analysed mathematically for deconvolution of the curves 
indicate that if the values are much lower than 2, the system is multilayer. For values near 2 the ratio of intensities of bands $I(2 \mathrm{D}) / I(\mathrm{G})$ is associated to a two-layer graphene material and for values of 2 and higher, it corresponds to a single layer material. Therefore, when analysing Fig. 8, it is possible to note two important aspects: first, all ratios $I(2 \mathrm{D}) / I(\mathrm{G})$ are lower to 2 , corresponding to a multilayer material and second, as temperature increases, the ratio $I(2 \mathrm{D}) / I(\mathrm{G})$ also increases, which suggests that the increase of temperature is beneficial as it promotes the obtention of graphene material of lesser layers and in consequence, purer. In fact, the difficulty to eliminate inorganic residue could be related to the lower number of layers, as it would be more selective in allowing the permeability of the particle.

In Fig. 9, it is possible to observe the results of the microstructure analysis carried out for both, the initial working sample (CTP) as well as the solids obtained at different working temperatures. It is noteworthy to mention that the shown images were selected in order to provide the most information as possible of the acquired particles.

Figure 9(a) and (b) picture the pitch from which the synthesis process was done. Both pictures make a comparison between normal and STEM modes from the microscope used. As it can be seen, in the particle is a dense solid with no porosities nor any indication of being an agglomerate, which differs when analysing Fig. 9(c) and
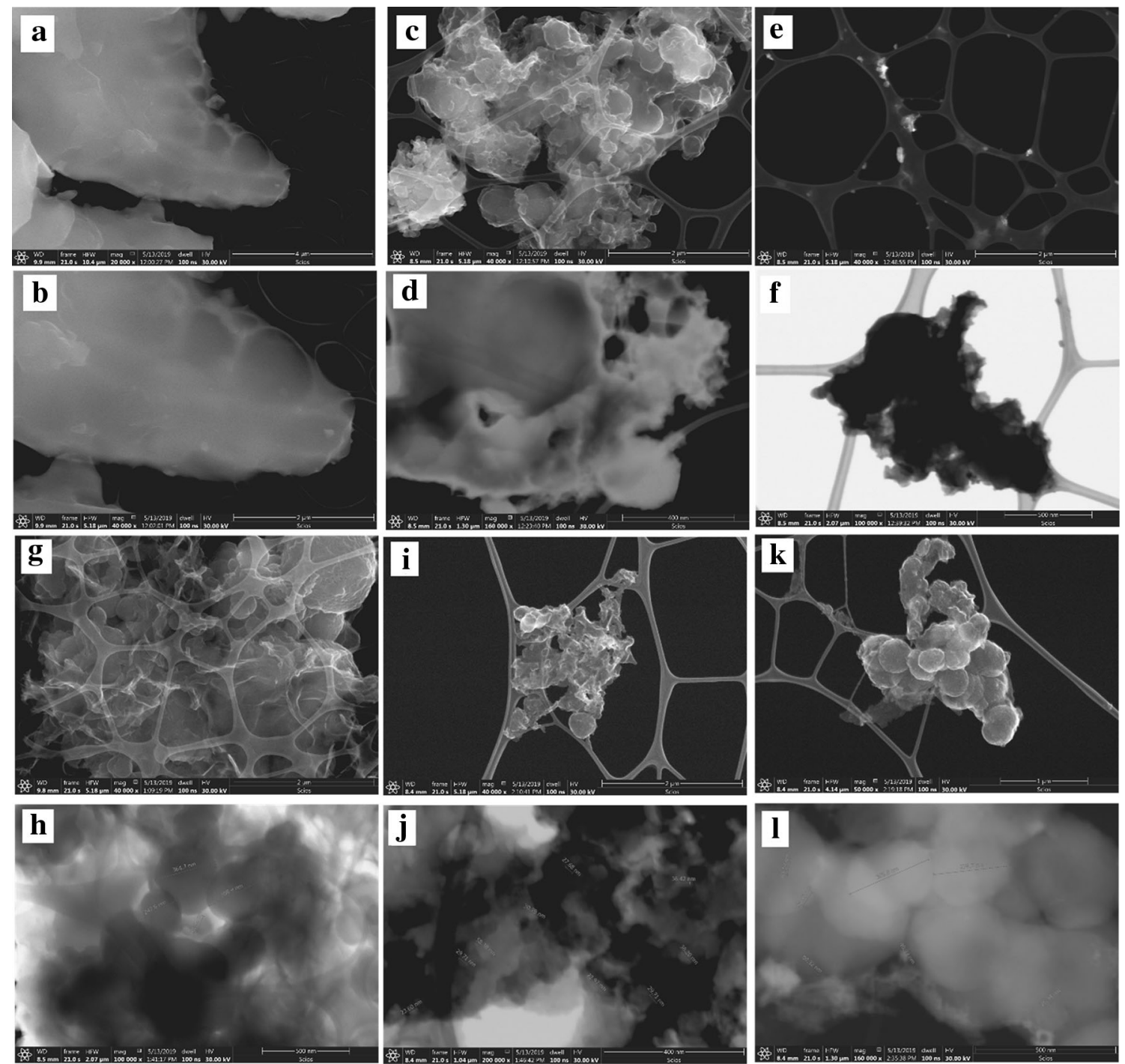

Fig. 9 a, b Initial CTP sample at a 40,000 $\times$ in normal and STEM mode, respectively; c obtained solids at $600{ }^{\circ} \mathrm{C}, 40,000 \times$ normal mode; d obtained solids at $600{ }^{\circ} \mathrm{C}, 160,000 \times$ STEM mode; e obtained solids at $700{ }^{\circ} \mathrm{C}, 40,000 \times$ normal mode; $\mathbf{f}$ obtained solids at $700{ }^{\circ} \mathrm{C}, 100,000 \times$ STEM mode; $\mathbf{g}$ obtained solids at $800{ }^{\circ} \mathrm{C}, 40,000 \times$ normal mode; $\mathbf{h}$ obtained solids at $800{ }^{\circ} \mathrm{C}, 100,000 \times$ STEM mode; $\mathbf{i}$ obtained solids at $850{ }^{\circ} \mathrm{C}, 40,000 \times$ normal mode; $\mathbf{j}$ obtained solids at $850{ }^{\circ} \mathrm{C}, 200,000 \times$ STEM mode; $\mathbf{k}$ obtained solids at $900{ }^{\circ} \mathrm{C}, 40,000 \times$ normal mode; $\mathbf{l}$ obtained solids at $900{ }^{\circ} \mathrm{C}, 160,000 \times$ STEM mode 


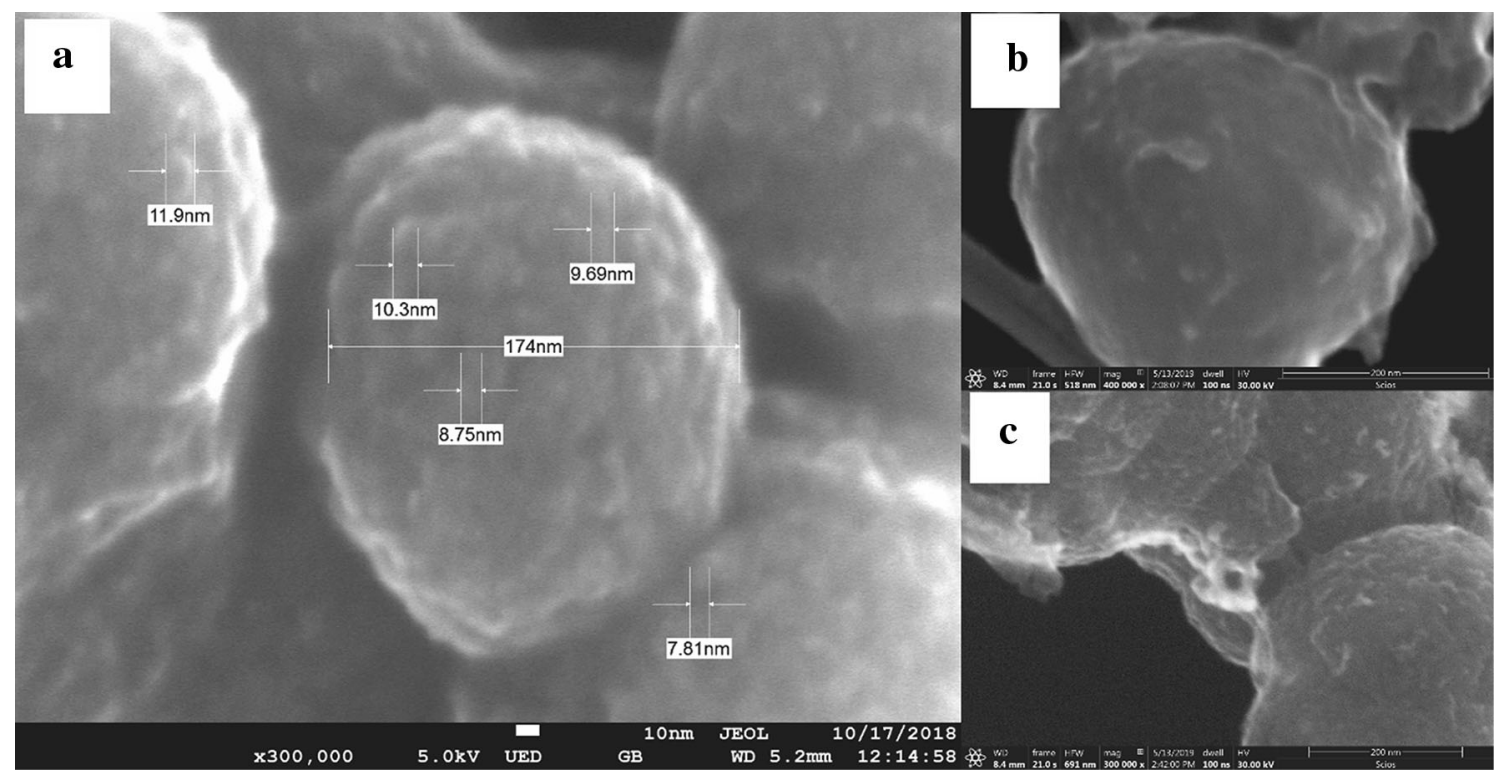

Fig. 10 Micrographs corresponding to obtained particles from synthesis at a $800{ }^{\circ} \mathrm{C}$ using FESEM at $300,000 \times ; \mathbf{b} 850{ }^{\circ} \mathrm{C}$ and $\mathbf{c} 900{ }^{\circ} \mathrm{C}$ utilising FEI 400,000 and $300,000 \times$ respectively

(d), where the synthesised solids at $600{ }^{\circ} \mathrm{C}$ are shown and where it is demonstrated that the particle, initially solid and without porosity, now has some kind of lumps, indicating the formation of GNCs. In fact, when comparing both images the STEM mode has a zone of the porous particle and another completely solid. In the other hand, the solids collected at $700{ }^{\circ} \mathrm{C}$ show dispersed particles and after analysing them under STEM mode, they reveal a more defined presence of nanometric capsules (Fig. 9e, f). However, when the solids at 800 (Fig. 9g) and $850{ }^{\circ} \mathrm{C}$ (Fig. 9i), it is possible to see well defined GNCs whose differences are in the size of the obtained particles, which can be confirmed by comparing the STEM images at both temperatures. At $800{ }^{\circ} \mathrm{C}$ there are particle sizes ranging from 200 and $500 \mathrm{~nm}$ (Fig. 9h) whereas at $850{ }^{\circ} \mathrm{C}$ the particle size varies between 20 and $40 \mathrm{~nm}$ in diameter (Fig. 9j). When increasing the temperature to $900{ }^{\circ} \mathrm{C}$, particles smaller than $100 \mathrm{~nm}$ are also obtained, as well as agglomerates between 200 and $300 \mathrm{~nm}$ (Fig. 9k, 1). The presence of such agglomerates can be confirmed with the analysis of Fig. 10, which also shows the formation of spherical particles comprised of nanometric particles. This phenomenon can be seen at 800,850 and $900{ }^{\circ} \mathrm{C}$, whose micrographs correspond to Fig. 10(a)-(c) respectively. For informative purposes, in Fig. 10(a), a particle with a diametre of $174 \mathrm{~nm}$ and comprised by particles of smaller size (approximately from 7 to $17 \mathrm{~nm}$ ) can be seen; in Fig. 10(b) and (c), it can be confirmed that at higher temperatures the phenomenon of agglomeration also presents itself.

\section{Conclusions}

(1) The analysis of the treated particles at 600 and $700{ }^{\circ} \mathrm{C}$ present difficulty in the removal of impurities, showing a crystalline behaviour in XRD. The microstructure analysis indicates that the formation of GNCs is not complete. The presence of inorganic material is observed in the results of Raman Spectroscopy.

(2) The particles with the best characteristics (when compared to literature) correspond to those treated at 800 and $850{ }^{\circ} \mathrm{C}$, analysed by XRD, Raman Spectrometry and microstructure analysis (spherical particles of nanometric size).

(3) At $900{ }^{\circ} \mathrm{C}$, it is possible to obtain a solid morphologically similar to those obtained and 800 and $850{ }^{\circ} \mathrm{C}$. However, according to the experimental results, a significant quantity of synthesised material is not obtained.

(4) In the analysis of Raman patterns by mathematical deconvolution, it can be shown that the ratio $I(2 \mathrm{D}) /$ $I(\mathrm{G})$ has an increase as the working temperature rises. However, after analysing the results, it can be said that working with temperatures between 800 and $850{ }^{\circ} \mathrm{C}$ continues to be the recommended temperature for a process applicable for industry (after a major study).

(5) It was confirmed that an agglomeration phenomenon appears when the particles are synthesised at temperatures ranging from 800 to $900{ }^{\circ} \mathrm{C}$. 
Acknowledgements The authors would like to thank the Consejo Nacional de Ciencia y Tecnología (CONACyT) for the support offered during the undertaking of this project. We also acknowledge the School of Metallurgy of the Universidad Autónoma de Coahuila for allowing the use of laboratory equipment to carry out the experimentation of this research work. Lastly, we would like to recognize B.Ch.E. Hugo Rendón for allowing the use of equipment for the analysis and characterisation of samples in the laboratory for Research and Development of Altos Hornos de México, S. A. B. de C. V.

Authors' contributions Dr. DMPS: Doing experimentation, analysis and interpretation of results and writing the manuscript; Dr. AEGC: Writing and reviewer of manuscript; BEJALC: Characterisation of sinthesised solids and result analysis; BEAPG: Characterisation of sinthesised solids and result analysis.

Funding Altos Hornos de México, S. A. B. de C. V.: Funding for tests and analysis of synthesised materials. Consejo Nacional de Ciencia y Tecnología (CONACyT): Funding under "Proyecto FORDECYT 273496".

Availability of data and materials The dataset(s) supporting the conclusions of this article is (are) included within the article (and its additional file(s)).

\section{Compliance with ethical standards}

Conflict of interests The authors declare that there were no conflict of interests which could have influenced or biased the presented work.

Ethical standards The experiments undertaken in this research comply fully with all current laws of Mexico.

Open Access This article is licensed under a Creative Commons Attribution 4.0 International License, which permits use, sharing, adaptation, distribution and reproduction in any medium or format, as long as you give appropriate credit to the original author(s) and the source, provide a link to the Creative Commons licence, and indicate if changes were made. The images or other third party material in this article are included in the article's Creative Commons licence, unless indicated otherwise in a credit line to the material. If material is not included in the article's Creative Commons licence and your intended use is not permitted by statutory regulation or exceeds the permitted use, you will need to obtain permission directly from the copyright holder. To view a copy of this licence, visit http://creativecommons. org/licenses/by/4.0/.

\section{References}

Alcolea-Sánchez C (2013) Obtención de grafeno mediante métodos químicos de exfoliación. Universidad Pontificada ICAI- ICADE
Comillas, Madrid, Tesis de Ingeniería Escuela Técnica Superior de Ingeniería

Bermejo J, Menédez R, Figueiras A, Granda M (1995) The role of low-molecular-weight components in the pyrolysis of pitches. Fuel 74:1792-1799

Fernández-García L, Álvarez P, Pérez-Mas AM, Blanco C, Santamaría R, Menédez R, Granda M (2017) Peculiarities of the production of graphene oxides with controlled properties from industrial coal liquids. Fuel 203:253-260

Granada M (2011) Breas: origen, composición y comportamiento en pirólisis. Bol. Grupo Español del Carbón 20:5-10

He X, Li X, Ma H, Han J, Zhang H, Yu C, Xiao N, Qiu J (2017a) ZnO template strategy for the synthesis of 3D interconnected graphene nanocapsules from coal tar pitch as supercapacitor electrode materials. J Powder Sources 340:183-191

He X, Li X, Wang J, Ma H, Zhang H, Xie Y, Xiao N, Qiu J (2017b) From diverse polycyclic aromatic molecules to interconnected graphene nanocapsules for supercapacitors. Microp Mesop Mater 245:73-81

Jibrael RI, Mohammed MKA (2016) Production of graphene powder by electrochemical exfoliation of graphite electrodes immersed in aqueous solution. Optik 127:6384-6389

Olszowska K, Pang J, Wrobel PS, Zhao L, Ta HQ, Liu Z, Trezebicka B, Bachmatiuk A, Rummeli MH (2017) Three-dimensional nanostructured graphene: synthesis and energy, environmental and biomedical applications. Synth Met 234:53-85

Rodríguez-González C, Vasilievna-Kharissova O (2008) Propiedades y aplicaciones del grafeno. Ingenierías 9(38):17-23

Saldivar-Larré CJ (2014) El grafeno. Propiedades y aplicaciones, Facultad de ciencias y tecnologías, Universidad Católica Nuestra Señora de Asunción, Asunción, Paraguay

Silva-Molina RYG (2016) Obtención de grafeno bicapa por cizallamiento estabilizados con bromuro de cetiltrimetilamonio y estudio experimental de propiedades de dispersión inelástica. Tesis de Licenciatura Universidad de Sonora, División de Ciencias Exactas y Naturales-Departamento de Física, Sonora, México

Tascón JMD (2007) Materiales de carbono: estructuras y formas. Óptica Pura y Aplicada 40(2):149-159

$\mathrm{Xu} \mathrm{H}$, Lin Q, Zhou T, Chen T, Lin S, Dong S (2014) Facile preparation of graphene nanosheets by pyrolysis of coal-tar pitch with the presence of aluminum. J Anal Appl Pyrol 110:481-485

Yoon S-M, Choi WM, Baik H, Shin H-J, Song I, Kwon M-S, Bae JJ, Kim H, Lee YH, Choi J-Y (2012) Synthesis of multilayer graphene balls by carbon segregation from nickel nanoparticles. ACS Nano 6(8):6803-6811

Zander M (1995) Aspects of coal tar chemistry/a review. Polycyclic Aromat Compd 7:209-211

Zhang L, Xu D, Gao J, Zhou S, Zhao L, Zhang Z (2017) Extraction and mechanism for the separation of neutral N-compounds from coal tar by ionic liquids. Fuel 194:27-35 